\title{
Functional lipidomics of oxidized products from polyunsaturated fatty acids
}

\author{
Guichardant M, Chen P, Liu M., Calzada C, Colas R, Véricel E, and Lagarde $\mathrm{M}^{1}$ \\ Université de Lyon, UMR 1060 / CarMeN, INSERM, INSA-Lyon, Univ-Lyon 1, INRA
}

Footnote: 1: Correspondence to Michel Lagarde, UMR 1060 / CarMeN, IMBL, INSA-Lyon, 20 Ave A. Einstein, 69621 Villeurbanne, France.

(michel.lagarde@insa-lyon.fr)

\begin{abstract}
Because of their high degree of unsaturation, polyunsaturated fatty acids (PUFA) in mammals, with mainly 18, 20 and 22 carbons, can easily be autooxidized, and converted into many oxidized derivatives and degradation products. This short review reports on some of those relevant to the evaluation of oxidative stress in situ. In addition, the enzyme-dependent oxygenation by both dioxygenases and monooxygenases is briefly reviewed by functional and/or metabolic categories, pointing out the structure variety and the analytical approaches.
\end{abstract}

\section{Key words}

Lipid peroxidation, Oxygenases, Lipid mediators, Lipid analysis

\section{Introduction}

Polyunsaturated fatty acids (PUFA) of nutritional interest are highly susceptible to oxidative stress, which leads to lipid peroxidation with degradation products from primary hydroperoxides. Besides non enzymatic lipid peroxidation, enzyme-induced peroxidation, e.g. cyclooxygenases and lipoxygenases, is a process that leads to hydroperoxides specifically transformed into a series of more stable metabolites called eicosanoids, docosanoids and even octadecanoids from C20, C22 and C18 PUFA, respectively.

Functional lipidomics may be seen as targeted lipidomics addressed to classes of lipids associated with specific types of function or biological system, e.g. eicosanoids in the vascular bed. This contrasts with the measurement of a limited number of markers associated with a physiological or pathophysiological situation. In this short review, the analysis of oxidized products from PUFA will be discussed in relation with their biological relevance.

\section{Autooxidation of PUFA}

It is well known that the autooxidation of PUFA leads to many metabolites that have been used as markers of the process (Catala, 2009). The mostly used one is malondialdehyde (MDA), which can also be produced through the cleavage of prostaglandin $(P G) \mathrm{H}_{2}$, releasing equimolecular amount of 12-hydroxy-heptadecatrienoic acid (HHT) (Hecker et al. 1987), making MDA an index of cyclooxygenase pathway as well. In addition to be a global index of lipid peroxidation, it can derive from non-lipid precursors such as carbohydrates, amino acids and DNA (Janero, 1990). Despite this lack of specificity, the advantage of its measurement in urine for instance is to provide an integrative and non invasive assessment of overall oxidative stress in the body (Guichardant et al. 1994).

In contrast to this lack of specificity, products such as isoprostanes and neuroprostanes are quite specific, perhaps too much as they represent the peroxidation of only two precursors, i.e. arachidonic acid (ARA) and docosahexaenoic acid (DHA), respectively (Roberts \& Fessel 2004). In addition, they are quite numerous with 64 different isoprostane isomers (Morrow \& Roberts, 1997). Among them, at least two isoprostanes exert biological activities. 8-iso-PGF ${ }_{2 \alpha}$ and 8-Iso-PGE ${ }_{2}$ exhibit vasoconstricting effects (Fukunaga et al. 1993), with the latter also affecting platelet aggregation (Lahaie et al. 1998 \& Longmire et al. 1994). On the other hand, neuroprostanes could be sensitive biomarkers of brain injury in response to oxidative stress, and then be relevant to neurodegenerative diseases (Montime et al. 2004). 
We have been interested in hydroxy-alkenals that may be issued from the whole series of omega- 3 or omega-6 PUFA, in contrast to isoprostanes and neuroprostanes that are products of ARA and DHA only. In that case, 4-hydroxy-hexenal (4-HHE) and 4-hydroxy-nonenal (4-HNE) are indices of omega-3 and omega-6 peroxidation (or distal peroxidation in the esterified fatty chain), respectively (Guichardant et al. 2006; Bacot et al. 2007) (Figure 1). It can however be argued that hydroxyalkenals are reactive enough to make covalent adducts with protein residues (on thiol and amine groups) as well as with amino-phospholipids and other bio-amines (Jürgens et al. 1990; Guichardant et al. 1998). Therefore, the measurement of 4-HHE and 4-HNE only represents the remaining free molecules. Yet, this argument is also valid for chemically stable isoprostanes, and neuroprostanes, that are very likely (although not reported) to be beta-oxidized as described for prostaglandins, and then found partly in urine as dinor and tetranor derivatives (Diczfalusy 1994). The advantage of measuring hydroxy-alkenals is the possibility to include in the same run of analysis the measurement of other homologues such as 4-hydroxy-dodecadienal (Figure 2) derived from the 12-lipoxygenase product of ARA, 12-HpETE (Bacot et al. 2007). These molecules have been measured in blood plasma (Calzada et al. 2010), and a non invasive measurement of their acidic metabolites (Figure 1), 4hydroxy-hexaenoic/nonenoic acids in urine is a possible alternative (Guichardant et al. 2006). In terms of biological effects, recent results show their cytotoxic activity (Pillon et al. 2010).

Hydroperoxy derivatives of PUFA can also be reduced into their stable counterparts by glutathionedependent peroxidase (Foster \& Sumar 1997), namely hydroxy derivatives, and then escape the spontaneous cleavage into hydroxy-alkenals. In that case, the various hydroxy-eicosatetraenoic acids (HETEs) from ARA and hydroxy-octadecadienoic acids (HODEs) from linoleic acid can easily be measured (Figure 3). As an example of clinical investigation, 9- and 13-HODE have been found as the main hydroxy derivatives present in LDL, and are significantly increased in LDL from type 2 diabetic patients compared to control LDL (Colas, 2010). However, it should be noted that HETEs and HODEs may also derive from the action of lipoxygenases/glutathione peroxidase, making these markers as indices of both autooxidation and enzyme-dependent oxygenation. The only difference between autooxidation product and lipoxygenase-derived products is the $\mathrm{R} / \mathrm{S}$ racemic configuration in the former and the $\mathrm{S}$ configuration in the latter. The biological function of HETEs and HODEs has been thoroughly reviewed (Spector et al. 1988).

\section{Dioxygenase-dependent oxygenated products}

The main dioxygenases involved in oxygenation of PUFA are cyclooxygenases and lipoxygenases.

The former enzymes lead to the unstable cyclic prostaglandin endoperoxides, PGH, from dihomogamma-linolenic, arachidonic and eicosapentaenoic acids, namely $\mathrm{PGH}_{1}, \mathrm{H}_{2}$ and $\mathrm{H}_{3}$, because the enzyme activity is associated with hydroperoxidase in the same protein (PGH synthase) (Smith 2008). A series of PGD, $E$ and $F_{\alpha}$ synthases convert PGHs into primary and ubiquitous PGs. More tissuespecific isomerases such as thromboxane (TX) and prostacyclin (PGI) synthases convert PGHs into TxAs and PGIs, further hydrolyzed into TxBs and 6-oxo-PGFs (Figure 4) due to their instability. Their biological activities are diverse and tissue-specific (Smith et al. 2000). It should be however noticed that $\mathrm{PGI}_{1}$ cannot be formed (Bunting et al. 1978) and $\mathrm{TxA}_{3}$ is hardly produced (Boukhchache \& Lagarde 1982). Dihomo counterparts of $\mathrm{PG}_{2} \mathrm{~S}$ can be produced from docosatetraenoic or adrenic acid (Sprecher 1982). It might be worth to measure all those products by GC-MS or LC-MS (Samuelsson et al. 1975; Deems et al. 2007) to get an overview of the cyclooxygenation of PG precursors.

Lipoxygenases are less substrate-specific than cyclooxygenases as they simply require a 1,4-cis,cispentadiene structure (Ewans \& Sprecher 1985). The most classical ones are depicted as 5-, 12- and 15-lipoxygenases, based on the number of carbon to be oxygenated in arachidonic acid as a substrate (Yamamoto 1989). To be extended at many other possible PUFA substrates, 12- and 15lipoxygenases could better be named omega- 9 and omega- 6 lipoxygenases, respectively. The primary products are hydroperoxy derivatives that are easily reduced into hydroxyl counterparts if the biological system of interest is well endowed with glutathione peroxidase activity (Figure 5). The 5lipoxygenase products of arachidonic acid, mainly leukotrienes, have been involved in many pathophysiological states, especially inflammation (Samuelsson, 1986). The scheme may however be much 
more complicated if we consider that primary products of PUFA may be further oxygenated if they contain the required additional double bonds (Serhan \& Samuelsson 1988). Because of substantial absorption molecular coefficient of conjugated dienes, trienes, even tetraenes which are always present in those products, LC separation with UV detection may sometimes be sensitive enough. When coupled with mass spectrometry, the LC-MS combination will increase the sensitivity and specificity (Poulsen et al. 2008).

More recently, numerous bioactive hydroxyl derivatives of DHA and eicosapentaenoic acid have been described by Serhan (2010), and called maresins, protectins and resolvins for their various activities in the anti-inflammatory processes. In addition, a double lipoxygenation of PUFA has been pointed out with a family of compounds that share platelet anti-aggregatory properties because of a conjugated trans,cis,trans $(\mathrm{E}, \mathrm{Z}, \mathrm{E})$ double bond geometry resulting from the double oxygenation (Chen et al. 2009 and 2011). These compounds, issued from various PUFA owning at least three double bonds at the omega- 6,9 and -12 positions, have been named poxytrins. They are dihydroxylated derivatives obtained by double lipoxygenation of PUFA, followed by reduction of the intermediate hydroperoxides. Although their biological relevance remains to be ascertained as endogenous products, their LC separation for a putative measurement in biological samples is to be considered in the same run together with the intermediates from single lipoxygenation, also reduced into mono-hydroxy derivatives. An example of separation of poxytrins and mono-hydroxy intermediates is given with a discrimination based on their $\lambda \max$ (Figure 6). Although most of the direct enzyme-dependent oxygenation leads to the $\mathrm{S}$ stereo-configuration, some $\mathrm{R}$ alcoholic carbons can be found, and the chromatographic step in the separation and evaluation is crucial, sometimes requiring chiral stationary phases.

\section{Monooxygenation of PUFA or derivatives}

Another important oxygenation pathway of PUFA is distinct from dioxygenase-dependent and enzyme-independent peroxidation by using monoxygenases that require cytochrome $\mathrm{P}_{450}$. Those monooxygenases may hydroxylate the substrate, making a primary (omega oxidation) or secondary alcohol, or making an epoxide at the expense of one double bond (Figure 7). Their biological relevance is less accurately stated, and this pathway for PUFA has not been so well developed compared to dioxygenase pathways. The omega hydroxylation has been sometimes described as a degradation pathway, as exemplified with leukotriene $B_{4}$ that is first transformed into 20-hydroxy$\mathrm{LTB}_{4}$ before being further oxidized into its 20 carboxy derivative which is entirely devoid of chemotactic activity (Shak \& Goldstein 1985). Omega or omega-1 hydroxylation has also been described with PUFA, especially arachidonic acid, to provide vasoactive derivatives (Capdevilla et al. 2000).

Another development has been made with the epoxidation of PUFA through cytochrome $P_{450}$ enzymes that have been called epoxygenases. The most popular products again are those issued from arachidonic acid (Capdevilla et al. 1992). The epoxy-eicosatrienoic acids (EET) formed are endowed of vasoactivities as well as their vicinal dihydroxy derivatives produced by specific epoxide hydrolases (Figure 7). The products are bioactive as well (Spector \& Norris 2007).

Most of those products being position isomers without any conjugated double bonds, their separation can be achieved by HPLC but their measurement cannot be done in proximal UV. One way of measuring the non-derivatized compounds is to use tandem mass spectrometry coupled with LC (Newman et al. 2002).

\section{Conclusion}

Eicosanoids as well as docosanoids and octadecanoids are so numerous, with still new compounds of biological interest to be described, that they represent a typical example of what the functional lipidomics is made for, i.e. describe and quantify as much as possible the different lipid species to get proper structure-function relationships, and evaluate their relevance in a given physiological or pathophysiological situation. As an exhaustive analysis is rarely possible, even when targeting a limited functional field, the measurement of an array of lipid metabolites in a specific field of interest 
allows to get the best balance sheet of the different pathways involved and bring information on their function.

\section{Acknowledgements}

The support of INSERM, Ministry of Education and Research, and LISA Carnot Institute is acknowledged.

\section{References}

Bacot, S., Bernoud-Hubac, N., Chantegrel, B., Deshayes, C., Doutheau, A., Ponsin, G., Lagarde, M., Guichardant, M., 2007. Evidence for in situ ethanolamine phospholipid adducts with hydroxyalkenals. J Lipid Res 48, 816-25.

Bunting, S., Moncada, S., Reed, P., Salmon, J.A., Vane, J.R., 1978. An antiserum to 5,6-dihydro prostacyclin (PGI1) which also binds prostacyclin. Prostaglandins 15, 565-73.

Boukhchache, D., Lagarde, M., 1982. Interactions between prostaglandin precursors during their oxygenation by human platelets. Biochim Biophys Acta 713, 386-92.

Calzada, C., Colas, R., Guillot, N., Guichardant, M., Laville, M., Véricel, E., Lagarde M., 2010. Subgram daily supplementation with docosahexaenoic acid protects low-density lipoproteins from oxidation in healthy men. Atherosclerosis 208, 467-72.

Capdevila, J.H., Falck, J.R., Estabrook, R.W., 1992. Cytochrome P450 and the arachidonate cascade. FASEB J 6,731-6.

Capdevila, J.H., Falck, J.R., Harris, R.C., 2000. Cytochrome P450 and arachidonic acid bioactivation. Molecular and functional properties of the arachidonate monooxygenase. J Lipid Res 41,63-81.

Catalá, A., 2009. Lipid peroxidation of membrane phospholipids generates hydroxy-alkenals and oxidized phospholipids active in physiological and/or pathological conditions. Chem Phys Lipids 157, $1-11$.

Chen, P., Fenet, B., Michaud, S., Tomczyk, N., Véricel, E., Lagarde, M., Guichardant, M., 2009. Full characterization of PDX, a neuroprotectin/protectin D1 isomer, which inhibits blood platelet aggregation. FEBS Lett 583, 3478-84.

Chen, P., Véricel, E., Lagarde, M., Guichardant, M., 2011. Poxytrins, a class of oxygenated products from polyunsaturated fatty acids, potently inhibit blood platelet aggregation. FASEB J 25, 382-8.

Colas, R., Pruneta-Deloche, V., Guichardant, M., Luquain-Costaz, C., Cugnet-Anceau, C., Moret, M., Vidal, H., Moulin, P., Lagarde, M., Calzada, C., 2010. Increased lipid peroxidation in LDL from type-2 diabetic patients. Lipids 45, 723-31.

Deems, R., Buczynski, M.W., Bowers-Gentry, R., Harkewicz, R., Dennis, E.A., 2007. Detection and quantitation of eicosanoids via high performance liquid chromatography-electrospray ionizationmass spectrometry. Methods Enzymol 432,59-82.

Diczfalusy, U., 1994. Beta-oxidation of eicosanoids. Prog Lipid Res 33, 403-28. 
Evans, R.W., Sprecher, H., 1985. Metabolism of icosa-5,11,14-trienoic acid in human platelets and the inhibition of arachidonic acid metabolism in human platelets by icosa-5,8,14-triynoic and icosa5,11,14-triynoic acids. Prostaglandins 29, 431-41.

Foster, L.H., Sumar, S., 1997. Selenium in health and disease: a review. Crit Rev Food Sci Nutr 37, 21128.

Fukunaga, M., Makita, N., Roberts, L., J. 2nd, Morrow, J., D., Takahashi, K., Badr, K., F., 1993. Evidence for the existence of F2-isoprostane receptors on rat vascular smooth muscle cells. Am J Physiol 264, C1619-24.

Guichardant, M., Bacot, S., Molière, P., Lagarde, M., 2006. Hydroxy-alkenals from the peroxidation of n-3 and n-6 fatty acids and urinary metabolites. Prostaglandins Leukot Essent Fatty Acids 75, 179-82.

Guichardant, M., Taibi-Tronche, P., Fay, L.B., Lagarde, M, 1998. Covalent modifications of aminophospholipids by 4-hydroxynonenal. Free Radic Biol Med. 25, 1049-56.

Guichardant, M., Valette-Talbi, L., Cavadini, C., Crozier, G., Berger, M., 1994. Malondialdehyde measurement in urine. J Chromatogr B Biomed Appl 655, 112-6.

Hecker, M., Haurand, M., Ullrich, V., Diczfalusy, U., Hammarström S., 1987. Products, kinetics, and substrate specificity of homogeneous thromboxane synthase from human platelets: development of a novel enzyme assay. Arch Biochem Biophys 254, 124-35.

Janero, D.R., 1990. Malondialdehyde and thiobarbituric acid-reactivity as diagnostic indices of lipid peroxidation and peroxidative tissue injury. Free Radic Biol Med 9, 515-40. Review.

Jürgens, G., Ashy, A., Esterbauer, H., 1990. Detection of new epitopes formed upon oxidation of lowdensity lipoprotein, lipoprotein (a) and very-low-density lipoprotein. Use of an antiserum against 4hydroxynonenal-modified low-density lipoprotein. Biochem J 265, 605-8.

Lahaie, I., Hardy, P., Hou, X., Hasséssian, H., Asselin, P., Lachapelle, P., Almazan, G., Varma, D.R., Morrow, J.D., Roberts, L.J. 2nd, Chemtob, S., 1998. A novel mechanism for vasoconstrictor action of 8-isoprostaglandin F2 alpha on retinal vessels. Am J Physiol 274, R1406-16.

Longmire, A.W., Roberts, L.J., Morrow, J.D., 1994. Actions of the E2-isoprostane, 8-ISO-PGE2, on the platelet thromboxane/endoperoxide receptor in humans and rats: additional evidence for the existence of a unique isoprostane receptor. Prostaglandins 48, 247-56.

Montine, K.S., Quinn, J.F., Zhang, J., Fessel, J.P., Roberts, L.J., 2nd, Morrow, J.D., Montine, T.J., 2004. Isoprostanes and related products of lipid peroxidation in neurodegenerative diseases. Chem Phys Lipids 128, 117-24.

Morrow, J.D., Roberts, L.J., 1997. The isoprostanes: unique bioactive products of lipid peroxidation. Prog Lipid Res 36, 1-21. Review.

Newman, J.W., Watanabe, T., Hammock, B.D., 2002. The simultaneous quantification of cytochrome P450 dependent linoleate and arachidonate metabolites in urine by HPLC-MS/MS. J Lipid Res 43, 1563-78. 
Pillon, N.J., Soulère, L., Vella, R.E., Croze, M., Caré, B.R., Soula, H.A., Doutheau, A., Lagarde, M., Soulage, C.O., 2010. Quantitative structure-activity relationship for 4-hydroxy-2-alkenal induced cytotoxicity in L6 muscle cells. Chem Biol Interact 188, 171-80.

Poulsen, R.C., Gotlinger, K.H., Serhan, C.N., Kruger, M.C., 2008. Identification of inflammatory and proresolving lipid mediators in bone marrow and their lipidomic profiles with ovariectomy and omega-3 intake. Am J Hematol 83, 437-45.

Roberts, L., J. 2nd, Fessel, J., P., 2004. The biochemistry of the isoprostane, neuroprostane, and isofuran pathways of lipid peroxidation. Chem Phys Lipids 128, 173-86.

Samuelsson, B., 1986. Leukotrienes and other lipoxygenase products. Prog Lipid Res 25, 13-8.

Samuelsson, B., Granström, E., Green, K., Hamberg, M., Hammarström, S., 1975. Prostaglandins. Annu Rev Biochem 44, 669-95.

Serhan, C.N., 2010. Novel lipid mediators and resolution mechanisms in acute inflammation: to resolve or not? Am J Pathol 177, 1576-91.

Serhan, C.N., Samuelsson B. Lipoxins: a new series of eicosanoids (biosynthesis, stereochemistry, and biological activities). Adv Exp Med Biol. 1988;229:1-14.

Shak, S., Goldstein, I.M., 1984. Omega-oxidation is the major pathway for the catabolism of leukotriene B4 in human polymorphonuclear leukocytes. J Biol Chem 259, 10181-7.

Smith, W.L., 2008. Nutritionally essential fatty acids and biologically indispensable cyclooxygenases. Trends Biochem Sci 33, 27-37.

Smith, W.L., DeWitt, D.L., Garavito, R.M., 2000. Cyclooxygenases: structural, cellular, and molecular biology. Annu Rev Biochem 69, 145-82.

Spector, A.A., Gordon, J.A., Moore, S.A., 1988. Hydroxyeicosatetraenoic acids (HETEs). Prog Lipid Res 27, 271-323.

Spector, A.A., Norris, A.W., 2007. Action of epoxyeicosatrienoic acids on cellular function. Am J Physiol Cell Physiol 292, C996-1012.

Sprecher, H., VanRollins, M., Sun, F., Wyche, A., Needleman, P., 1982. Dihomo-prostaglandins and thromboxane. A prostaglandin family from adrenic acid that may be preferentially synthesized in the kidney. J Biol Chem 257, 3912-8.

Yamamoto, S., 1989. Mammalian lipoxygenases: molecular and catalytic properties. Prostaglandins Leukot Essent Fatty Acids 35, 219-29.

\section{Figure legends}

Figure 1: Distal peroxidation and subsequent degradation by cleavage of the cis (Z) double bond of the conjugated diene leading to a hydroxyl-alkenal. 4-hydroxy-2E-nonenal (4HNE) and 4-hydroxy-2Ehexenal (4-HHE) derives from omega-6 and omega-3 PUFA, respectively. Further oxidation of the hydroxyl-alkenal leads to corresponding carboxylic acids, 4-hydroxy-2E-nonenoic (4-HNA) and 4hydroxy-2E-hexenoic (4-HHA) acids. 
Figure 2: 12 -lipoxygenation of an omega-6 polyunsaturated fatty acid (PUFA), such as arachidonic acid, and subsequent degradation/cleavage leading to 4-hydroxy-2E-dodecadienal (4-HDDE), further oxidized into 4-hydroxy-2E-dodecadienoic acid (4-HDDA).

Figure 3: Reverse phase HPLC profile of monohydroxy-derivatives of linoleic (HODEs) and arachidonic (HETEs) acids of biological relevance, with detection at $235 \mathrm{~nm}$. Sensitivity can be evaluated from the less represented metabolite, $15-\mathrm{HETE}$, as the signal corresponds to around $10 \mathrm{pmol}$.

Figure 4: Prostanoid generation from arachidonic acid. $\mathrm{PGG}_{2}$ and $\mathrm{PGH}_{2}$ from arachidonic acid are shown as an example of what may occur from dihomogammalinolenic and eicosapentaenoic acids, with the production of $P G_{1} / H_{1}$ and $P G G_{3} / H_{3}$, respectively. The formation of the other prostanoids also occurs from the latter two PUFA, except for $\mathrm{PGI}_{1}$ that cannot be formed because of the lack of double bond at carbon 5 . The same metabolic pathways have been reported for dihomo-arachidonic or adrenic acid with the products called dihomo-prostanoids.

PG: prostaglandin; Tx: thromboxane; PG-S: PG synthase; PGH-S is a bi-functional enzyme protein with two activities: cyclooxygenase producing PGG, and hydroperoxidase converting PGG into PGH.

Figure 5: 5-lipoxygenase pathway from arachidonic acid leading to leukotriene (LT) $A_{4}$, further converted into $\mathrm{LTB}_{4}$ or $\mathrm{LTC}_{4}$, the first peptido-LTs. Part of 5 -HpETE is reduced into 5-HETE by glutathione peroxidase. $\mathrm{LT}_{5 \mathrm{~s}}$ can be obtained from eicisapentaenoic acid, and $\mathrm{LTB}_{3}$ can be produced from 5,8,11-eicosatrienoic acid (20:3w-9 or Mead acid).

Lox: lipoxygenase; H(p)ETE: hydroxy(peroxy)-eicosatetraenoic acid; GSH: reduced glutathione.

Figure 6: Reverse phase HPLC profiles of monohydroxy- and dihydroxy-derivatives initiated by 15/w6lipoxygenase acting upon various PUFA having at least three $Z$ double bonds from the omega- 6 position. The dihydroxy-derivatives contain an E,Z,E conjugated motif with the E,Z,E geometry. These derivatives have been collectively named poxytrins (PUFA oxygenated trienes). The monohydroxy, and dihydroxy-derivatives have been detected at 235 and $270 \mathrm{~nm}$, respectively.

Figure 7: Metabolic pathways of arachidonic acid through cytochrome $\mathrm{P}_{450}$ monooxygenases. Cyt: cytochrome; EET: epoxy-eicosatrienoic acid; DHETE: dihydroxy-eicosatrienoic acid. 